\title{
Conhecimento e Uso de Plantas Medicinais por Usuários de Unidades Básicas de Saúde na Região de Colombo, PR
}

\author{
The knowledge and use of medicinal plants by users of primary healthcare \\ facilities in Colombo, PR
}

\author{
VINÍCIUS BEDNARCZUK DE OLIVEIRA ${ }^{1}$ \\ THAIS REGINA MEZZOMO² \\ ELIÉZER FERNANDA DE MORAES ${ }^{3}$
}

\section{RESUMO}

Objetivo: Avaliar o conhecimento sobre a utilização de plantas medicinais pela população frequentadora de Unidades Básicas de Saúde (UBS) em Colombo, PR. Material e Métodos: Por meio de um questionário de pesquisa estruturado, foram entrevistados usuários adultos de ambos os sexos, de quatro UBS, entre os meses de maio e junho de 2016. Resultados: Foram entrevistados 101 usuários de UBS. Destes, 72,28\% utilizavam plantas medicinais, sendo a maioria do sexo feminino, com idade média de 39 anos. A espécie vegetal mais citada pela população foi a camomila (Matricaria chamomilla L.), seguida da hortelã (Mentha spicata L.) e do capim limão (Cymbopogon citratus L.), sendo que a indicação mais referida pelos entrevistados foi calmante, seguida das afecções estomacais. Aproximadamente $70 \%$ dos entrevistados que fazem uso de plantas medicinais relataram que cultivam as próprias plantas para consumo e o entendimento da finalidade terapêutica é proveniente do conhecimento familiar. Dentre as 30 plantas citadas pelos entrevistados, grande parte estão listadas na Relação Nacional de Plantas Medicinais de Interesse ao SUS (RENISUS), no Formulário Fitoterápico e no Memento Fitoterápico da Farmacopeia Brasileira. Conclusão: A partir dos resultados obtidos, conclui-se que os usuários entendem que as plantas medicinais auxiliam na terapêutica como um complemento no tratamento das mais diversas enfermidades.

\section{DESCRITORES}

Fitoterapia. Plantas Medicinais. Terapias Complementares. Saúde Pública.

\begin{abstract}
Objective: To evaluate the knowledge on the use of medicinal plants by frequent users of Primary Healthcare Facilities (PHF) in Colombo, PR. Material and Methods: Adult users of both sexes from four PHF were interviewed using a structured questionnaire between May and June 2016. Results: A total of 101 PHF users were interviewed. Of these, $72.28 \%$ reported using medicinal plants, most of them being female, with a mean age of 39 years. The plant species most frequently cited were chamomile (Matricaria chamomilla L.), followed by mint (Mentha spicata L.) and lemon grass (Cymbopogon citratus L.), and the most common indications were soothing, followed by stomach disorders. Approximately $70 \%$ of the respondents who use medicinal plants reported that they cultivate the plants themselves for consumption, and that the knowledge of the therapeutic purpose comes from the family. Among the 30 plants cited by respondents, most of them are listed in the National List of Medicinal Plants of Interest to the Brazilian Healthcare System (SUS, RENISUS); in the Phytotherapic Form; and in the Phytotherapic Memento of the Brazilian Pharmacopoeia. Conclusion: It is concluded that users understand that medicinal plants help in therapy as an adjunct in the treatment of several diseases.
\end{abstract}

\section{DESCRIPTORS}

Phytotherapy. Medicinal plants. Complementary Therapies. Public Health.

\footnotetext{
Doutor em Ciências Farmacêuticas pela Universidade Federal do Paraná - UFPR. Docente do Centro Universitário Campos de Andrade UNIANDRADE, Curitiba, PR, Brasil.

2 Mestre em Segurança Alimentar e Nutricional pela Universidade Federal do Paraná - UFPR. Docente da Universidade Positivo, Curitiba, PR, Brasil.

3 Nutricionista pelo Centro Universitário Campos de Andrade - UNIANDRADE, Curitiba, PR, Brasil.
} 
egundo dados da Organização Mundial da Saúde (OMS), 80\% da população de países em desenvolvimento utilizam a medicina tradicional exclusivamente como prática na atenção primária à saúde e, deste total, $85 \%$ fazem uso de plantas medicinais e de extratos vegetais ${ }^{1}$. A utilização de plantas medicinais no Brasil possui alguns facilitadores, como a grande diversidade vegetal e o baixo custo associado à terapêutica, fatores que despertam a atenção dos programas de assistência à saúde e profissionais² ${ }^{2}$.

A fitoterapia é um método de tratamento caracterizado pela utilização de plantas medicinais em suas diversas preparações, constituindo uma modalidade de terapia integrativa e complementar diante das necessidades de saúde. Seu uso tem sido crescente na população de diversos países ${ }^{3,4}$. No Brasil existem duas políticas nacionais que incentivam a implementação desta terapia. Criadas em 2006, a Política Nacional de Plantas Medicinais e Fitoterápicos (PNPMF) ${ }^{5}$ e a Política Nacional de Práticas Integrativas e Complementares (PNPIC) ${ }^{6}$, incrementaram a discussão sobre a oportunidade, a importância, as dificuldades, as facilidades e as vantagens da implementação da Fitoterapia nos Serviços de Saúde do Sistema único de Saúde (SUS). Além destas duas políticas, a Relação Nacional de Plantas Medicinais de Interesse ao SUS (RENISUS) apresenta uma lista com 71 espécies vegetais com potencial para gerar fitoterápicos ao SUS $^{7}$, e, atualmente, a RDC 26/2014 divide os produtos derivados de plantas em duas categorias, sendo a primeira sobre os medicamentos fitoterápicos que devem apresentar segurança e eficácia através de ensaios clínicos e, por segundo, os produtos tradicionais fitoterápicos que poderão ser registrados através da comprovação da tradicionalidade ${ }^{8}$.

Diversos estudos afirmam que o uso de plantas medicinais está relacionado à cultura popular a qual é transmitida de geração para geração nas comunidades tradicionais ${ }^{9}$. De acordo com Santos e colaboradores ${ }^{2}$, não se deve subestimar o conhecimento empírico, já que muitos dos conhecimentos atuais dos efeitos das espécies vegetais conhecidos são oriundos deste conhecimento. Entretanto, vale ressaltar que diversas plantas apresentam efeitos tóxicos, e que a falsa ideia de que tudo que é natural é inócuo, precisa ser revista e conscientizada pela equipe multidisciplinar de Saúde prevenindo assim casos de intoxicação que podem levar a óbito ${ }^{10}$.

Os agentes de saúde são os profissionais qualificados e responsáveis por diagnosticar e indicar o tratamento adequado para as mais diversas patologias ${ }^{4,11}$. Em 17 de junho de 2016, foi aprovada a resolução RDC $N^{\circ} 84$ que aprovou o Memento Fitoterápico, um dos Compêndios da Farmacopeia Brasileira que traz as informações úteis para a prescrição de fitoterápicos ${ }^{12}$.

O Ministério da Saúde brasileiro tem estabelecido políticas que encorajam o desenvolvimento de estudos com plantas medicinais com o objetivo de evitar efeitos irracionais e danos que possam ser causados pelo mau uso desta modalidade terapêutica, além de colocar em prática os benefícios comprovados ${ }^{13}$. Nesta perspectiva, este trabalho teve como objetivo avaliar o conhecimento e o uso de plantas medicinais pela população frequentadora de Unidades Básicas de Saúde em Colombo, PR.

\section{METODOLOGIA}

Trata-se de um estudo do tipo observacional transversal aprovado pelo Comitê de Ética em Pesquisa (CEP) do Centro Universitário Campos de Andrade - UNIANDRADE, de Curitiba, PR sob o $n^{\circ}$ 53622915.7.0000.5218.

O município de Colombo, na região metropolitana de Curitiba, PR, possui 21 Unidades Básicas de Saúde (UBS). Este estudo foi realizado em quatro UBS, entre os meses de maio e junho de 2016, com indivíduos maiores de 18 anos, de ambos os sexos, que consentiram e assinaram o Termo de Consentimento Livre e Esclarecido (TCLE).

Os dados foram coletados através de um questionário composto por questões subjetivas e objetivas, adaptado de Ghizi e Mezzomo ${ }^{14}$. Esse questionário foi dividido em três partes, sendo que a primeira parte envolveu dados sobre a identificação do indivíduo como idade, sexo e região de localidade; a segunda parte referiu-se à avaliação econômica; e a terceira parte compreendeu o assunto sobre o uso de plantas medicinais. Os nomes científicos das plantas referidas foram definidos de acordo com Panizza, Veiga e Almeida 
$(2012)^{15}$. Após análise dos resultados, os dados obtidos foram contabilizados em média e desvio padrão no programa Microsoft Excel®.

\section{RESULTADOS}

A população pesquisada no presente trabalho constitui-se de 101 indivíduos de ambos os sexos usuários das UBS de Colombo, PR. A distribuição das variáveis sociodemográficas pode ser visualizada no Quadro 1.

As informações sobre a utilização de plantas medicinais pela população avaliada podem ser visualizadas no Quadro 2. Ressalta-se que cada respondente citou mais de uma espécie utilizada, totalizando 30 diferentes espécies.

Entre as finalidades de uso das diversas espécies vegetais, as que mais se destacaram foram a utilização como calmante $(34,38 \%)$, para afecções estomacais $(21,88 \%)$ e para gripe e resfriados $(7,81 \%)$.

Também foram avaliadas as plantas mais indicadas pelos diversos grupos identificados. Nas indicações realizadas pelos amigos, a espécie mais indicada foi a camomila (Matricaria chamomilla L.) (30\%) seguida do capim limão (Cymbopogon citratus L.) (20\%). Entre os familiares, $19,05 \%$ indicaram a camomila (M. chamomilla L.), seguida das espécies hortelã (Mentha spicata L.) e melissa (Melissa officinalis L.), sendo que ambas apresentaram 9,52\% de indicação. $\mathrm{Na}$ indicação médica, a planta mais indicada foi a hortelã $(M$. spicata L.) $(25 \%)$, seguido da camomila ( $M$. chamomilla L.) (20\%).

O Quadro 3 demonstra a finalidade de uso e a origem das indicações da camomila $(M$. chamomilla L.), principal planta medicinal utilizada pela população entrevistada.

Quando questionados os usuários das UBS se eles sabiam que as plantas medicinais possuem restrição de uso, apenas $26,56 \%$ revelaram que tinham conhecimento de que as plantas medicinais podem causar efeitos deletérios. Destaca-se que, dentro da população que afirmou conhecer os riscos, crianças e gestantes foram apontadas como o grupo de maior risco.

\begin{tabular}{|c|c|}
\hline \multicolumn{2}{|c|}{$\begin{array}{l}\text { QUADRO } 1 \text { - Caracterização sociodemográfica de } \\
\text { usuários de Unidades Básicas de Saúde de } \\
\text { Colombo. PR. }\end{array}$} \\
\hline Variáveis & $n^{\circ}(\%)$ \\
\hline $\mathrm{n}^{\circ}$ de indivíduos & $101(100 \%)$ \\
\hline Sexo & $n^{\circ}(\%)$ \\
\hline Feminino & $58(57,42 \%)$ \\
\hline Masculino & $43(42,58 \%)$ \\
\hline Escolaridade & $n^{\circ}(\%)$ \\
\hline Analfabeto & $9(8,91)$ \\
\hline Ensino Fundamental Completo & $26(25,74)$ \\
\hline Ensino Fundamental Incompleto & $7(6,93)$ \\
\hline Ensino Médio & $49(48,51)$ \\
\hline Ensino Superior Completo & $8(7,93)$ \\
\hline Ensino Superior Incompleto & $2(1,98)$ \\
\hline Profissão & $n^{\circ}(\%)$ \\
\hline Vendedor & $18(17,82)$ \\
\hline Dona de Casa & $17(16,83)$ \\
\hline Agricultor & $13(12,87)$ \\
\hline Aposentado & $10(9,90)$ \\
\hline Motorista & $10(9,90)$ \\
\hline Outros & $33(32,68)$ \\
\hline Profissão & Média \pm DP \\
\hline Idade & 39,34 anos $\pm 15,61$ \\
\hline Renda & Média \pm DP \\
\hline Renda & $\mathrm{R} \$ 780,34 \pm 379,70$ \\
\hline
\end{tabular}


QUADRO 2 - Utilização e indicação de uso de plantas medicinais por usuários de Unidades Básicas de Saúde de Colombo, PR.

\begin{tabular}{lc}
\multicolumn{1}{c}{ Fatores Analisados } & $\%$ \\
Utiliza Plantas Medicinais & 72,28 \\
Não Utiliza Plantas Medicinais & 27,72 \\
Plantas Utilizadas & \\
Camomila (Matricaria chamomilla L.) & 20,31 \\
Hortelã (Mentha spicata L.) & 10,94 \\
Capim Limão (Cymbopogon citratus L.) & 6,25 \\
Melissa (Melissa officinalis L.) & 6,25 \\
Erva Doce (Foeniculum vulqare Mill.) & 5,47 \\
Tanchaqem (Plantaqo maior L.) & 5,47 \\
Erva Cidreira (Lippia alba Mill.) & 4,69 \\
Cavalinha (Equisetum hvemale L.), \\
Folha de Larania (Citrus sinensis L.), \\
Genqibre (Zinqiber officinale Roscoe), \\
Outros Forma de Preparo & 3,91 \\
& 3,91 \\
Decoccão & 3,91 \\
Infusão & 28,89 \\
Maceração & \\
& 29,69 \\
Casa de Produtos Naturais & 69,53 \\
Farmácia & 0,78 \\
Quintal & \\
Amiqos Indicação & 27,34 \\
Familiares & 3,13 \\
Familiares e Amiqos & 69,53 \\
Médico & 7,81 \\
& 65,63 \\
& 10,94 \\
& 15,63
\end{tabular}

\begin{tabular}{|c|c|}
\hline \multicolumn{2}{|c|}{$\begin{array}{c}\text { Quadro } 3 \text { - Finalidade de uso da Camomila (Matricaria chamomilla L.) e } \\
\text { origem de suas indicações segundo usuários de Unidades Básicas de Saúde } \\
\text { de Colombo, PR. }\end{array}$} \\
\hline Variáveis & $N^{\circ}(\%)$ \\
\hline \multicolumn{2}{|l|}{ Indicado por Amigos como: } \\
\hline Calmante & $3(11,54)$ \\
\hline \multicolumn{2}{|l|}{ Indicado por Familiar como: } \\
\hline Antiemético & $1(3,85)$ \\
\hline Calmante & $9(34,62)$ \\
\hline Calmante e para afecções estomacais & $2(7,69)$ \\
\hline Afecções estomacais & $2(7,69)$ \\
\hline Em substituição do café & $2(7,69)$ \\
\hline \multicolumn{2}{|l|}{ Indicado por Familiar, amigos como: } \\
\hline Calmante & $3(11,54)$ \\
\hline \multicolumn{2}{|l|}{ Indicação Médica como: } \\
\hline Calmante & $4(15,38)$ \\
\hline
\end{tabular}




\section{DISCUSSÃO}

Em relação aos dados encontrados neste estudo, observou-se que a prevalência de utilização de plantas medicinais $(72,28 \%)$ foi em indivíduos do sexo feminino com idade inferior a 40 anos, com baixa renda e escolaridade. Resultados semelhantes aos encontrados em outros estudos com temática semelhante ${ }^{16,17}$.

Observou-se que o uso de plantas medicinais, na maioria das vezes, não foi indicado por um profissional habilitado, mas sim, por meio do conhecimento empírico passado de geração em geração pelos familiares. Estes resultados são semelhantes a outros estudos, demonstrando que as pessoas mais idosas possuem maior conhecimento sobre o uso de plantas medicinais ${ }^{18}$.

Sobre a renda per capta dos entrevistados, enquadram-se na classe econômica $D-E$, sendo o resultado inferior a um salário mínimo, que atualmente é de $\mathrm{R} \$ 880,00$ reais $^{19}$. Muito estudos citam que o uso de plantas medicinais é realizado por pessoas que apresentam uma renda mais baixa, buscando assim uma "alternativa econômica" no tratamento de enfermidades, por não apresentarem condições de compra de medicamentos ${ }^{14,20}$.

Com relação às 30 variedades de plantas mencionadas pela população pesquisada, 20 delas estão presentes no Formulário Nacional Fitoterápico ${ }^{21}$, com modo de preparo, indicações e contraindicações sobre o uso da espécie vegetal. Já no RENISUS ${ }^{7}$ e no Memento Fitoterápico da Farmacopeia Brasileira ${ }^{12}$, apenas 13 e 10 plantas, respectivamente, estão contempladas.

A finalidade que mais relatada sobre o uso de plantas medicinais foi para o tratamento como calmante. De acordo com as respostas obtidas, as plantas utilizadas para esta finalidade foram: camomila (Matricaria chamomilla L.), capim limão (Cymbopogon citratus L.), erva cidreira (Lippia alba Mill.), erva doce (Foeniculum vulgare Mill.), hortelã (Mentha spicata L.) e melissa (Melissa officinalis
L.) e, em menor quantidade, alecrim (Rosmarinus officinalis L.), erva de São João (Hypericum perforatum L.), folha de maracujá (Passiflora sp.). De acordo com o Formulário Nacional Fitoterápico a camomila (M. chamomilla L.), o capim limão ( $C$. citratus L.), a erva cidreira ( $L$. alba Mill.), a folha de maracujá (Passiflora sp.) e melissa (M. officinalis L.) apresentam e são utilizadas para essa finalidade. Porém, algumas das espécies citadas pelos entrevistados para esta mesma finalidade não possuem esta indicação, tais como: alecrim (Rosmarinus officinalis L.), erva doce (F. vulgare Mill.) e hortelã (M. spicata L. $)^{21}$. A erva de São João (H. perforatum L.) apresenta função ansiolítica, porém não encontra-se listada nesse formulário ${ }^{22} \mathrm{e}$ segundo a Instrução Normativa $n^{\circ} 5$ de 2008 que determina a Lista de Medicamentos Fitoterápicos de Registro Simplificado, esta planta sob a forma de medicamento fitoterápico deve ser vendida apenas sob prescrição médica ${ }^{23}$. Dentre as plantas citadas, a grande maioria apresenta efeitos sedativos e ansiolíticos leves ${ }^{15}$.

Verificou-se que algumas plantas são utilizadas erroneamente pela população pesquisada como, por exemplo, a utilização de plantas para substituir o café. As plantas relatadas para esta finalidade foram camomila ( $M$. chamomilla $L$.) e erva cidreira ( $L$. alba Mill.), porém, sabe-se que o café possui ação estimulante do sistema nervoso central e, as espécies relatadas, possuem efeito antagônico. Resultados semelhantes sobre o uso controverso de plantas medicinais já foram relatados ${ }^{24}$.

Sobre as formas de preparo, três modos foram citados pelos entrevistados, sendo: método de infusão, decocção e maceração. O método de infusão apresentou a maior prevalência. Resultados semelhantes já foram apontados por Lima e colaboradores ${ }^{25}$, porém ressalta-se que alguns métodos não são os indicados pelas literaturas especializadas ${ }^{12,21}$, como exemplo as plantas camomila ( $M$. chamomilla L.), capim limão ( $C$. 
citratus L.), erva doce ( $F$. vulgare Mill.) e hortelã ( $M$. spicata L.) que quando preparadas por decocção podem perder óleo essencial, constituinte químico responsável também pelos efeitos terapêuticos, reduzindo assim sua ação.

A forma de aquisição da matéria prima vegetal é outro fator que precisa ser levado em consideração. Dos entrevistados, $69,53 \%$ cultivam as plantas em casa, resultado que corrobora com diversos estudos ${ }^{24,25}$. Neste sentido, destaca-se a importância do conhecimento botânico para identificação correta da espécie vegetal cultivada, pois seu uso errôneo pode diminuir o efeito terapêutico, ou não apresentar o efeito esperado, e em casos de plantas com potencial tóxico, pode desencadear a intoxicação ou até mesmo levar o usuário da planta a óbito ${ }^{3,24,25}$.

Dados do Sistema de Informações TóxicoFarmacológicas (SINITOX) de 2013 apontam que a intoxicação por plantas representa $1,05 \%$ dos casos de intoxicação no País, valores baixos quando comparados com medicamentos (28,45\%). Porém, grande parte dos casos de intoxicação por plantas não é notificado pelas autoridades ${ }^{26,27}$. O conhecimento sobre a espécie vegetal a ser utilizada e sua indicação de uso pela população que utiliza estas espécies é fundamental, pois gestantes, crianças, cardiopatas, entre outros precisam de cuidados ao consumir evitando assim abortos, efeitos teratogênicos, infarto, intoxicações e até mesmo o óbito ${ }^{26,28}$.

Considerando o estilo de vida estressante da sociedade atual, a alimentação inadequada de grande parte da população e as prevalências de depressão em diferentes idades, culminando muitas vezes em aposentadorias por invalidez, os resultados desse estudo demonstram que a população estudada procura o alívio para estas afecções através do uso de terapias naturais ${ }^{14,22,24}$.

O conhecimento dos profissionais de saúde sobre as generalidades que envolvem este tema, até a prescrição e conhecimento dos efeitos que as espécies vegetais possuem, é de fundamental importância para que o uso de plantas medicinais seja levado a sério, desmistificando o ditado popular de que "o que é natural é inócuo".

O Memento Fitoterápico da Farmacopeia Brasileira ${ }^{12}$ visa colaborar com este conhecimento tradicional aos profissionais de saúde prescritores, orientando através de evidências científicas a prescrição de plantas medicinais e fitoterápicos.

O uso da fitoterapia como tratamento complementar no atendimento primário à saúde é de fundamental importância ao SUS, contribuindo como uma ferramenta de baixo custo econômico, podendo diminuir os casos de internamento com a tradicionalidade do uso de plantas medicinais pela população.

\section{CONCLUSÃO}

Diante dos resultados apresentados, concluise que mais de $70 \%$ dos usuários das Unidades Básicas de Saúde da região de Colombo - PR fazem uso de plantas medicinais, sendo a população composta predominantemente por usuários do sexo feminino, com idade média de 39 anos, com baixa escolaridade e renda per capta inferior a um salário mínimo. Estes usuários fazem uso de plantas medicinais principalmente para situações de ansiedade, e as plantas mais relatadas foram a camomila (Matricaria chamomilla L.), o capim limão (Cymbopogon citratus L.), e a melissa (Melissa officinalis L.). Ressalta-se que as autoridades governamentais têm criado programas e políticas que contribuem com a temática e, reforça-se a importância do profissional de saúde com conhecimento na prescrição de plantas medicinais e fitoterápicos para evitar o uso indevido e errôneo pela população podendo, inclusive, diminuir os casos de intoxicação e colaborar com o uso tradicional. 


\section{REFERÊNCIAS}

1. Bruning $M C R$, Mosegui $G B G$, Vianna $C M d M$. A utilização da fitoterapia e de plantas medicinais em unidades básicas de saúde nos municípios de Cascavel e Foz do Iguaçu - Paraná: a visão dos profissionais de saúde. Ciênc Saúde Coletiva. 2012;17(10):2675-85.

2. Santos RL, Guimaraes GP, Nobre MSC, Portela AS. Análise sobre a fitoterapia como prática integrativa no Sistema Único de Saúde. Rev Bras Plantas Med. 2011;13(4):486-91.

3. Figueredo CAD, Gurgel IGD, Gurgel Junior GD. A Política Nacional de Plantas Medicinais e Fitoterápicos: construção, perspectivas e desafios. Physis. 2014;24(2):381-400.

4. Maciel MAM, Pinto AC, Veiga JV, Grynberg NF, Echevarria

A. Plantas medicinais: a necessidade de estudos multidisciplinares. Quím Nova. 2002;25(3):429-38.

5. BRASIL. Ministério da Saúde. Secretaria de Ciência, Tecnologia e Insumos Estratégicos, Departamento de Assistência Farmacêutica. Política Nacional de Plantas Medicinais e Fitoterápicos. Brasília: Ministério da Saúde, 2006.

6. BRASIL. Ministério da Saúde. Secretaria de Assistência à Saúde. Departamento de Atenção Básica. Política Nacional de Práticas Integrativas e Complementares no SUS: PNPIC-SUS. Brasília: Ministério da Saúde, 2006.

7. BRASIL. Ministério da Saúde. RENISUS. Relação nacional de plantas medicinais de interesse ao SUS. Espécies vegetais, 2009.

8. BRASIL. Ministério da Saúde. Agência Nacional de Vigilância Sanitária. Resolução da Diretoria Colegiada RDC n²6, de 13 de maio de 2014.

9. Oliveira ER, Menini Neto L. Levantamento etnobotânico de plantas medicinais utilizadas pelos moradores do povoado de Manejo, Lima Duarte - MG. Rev Bras Plantas Med. 2012;14(2):311-20.

10. Bednarczuk VO, Verdam MCS, Miguel MD, Miguel OG. Testes in vitro $e$ in vivo utilizados nas triagem toxicológica de produtos naturais. Visão Acadêmica. 2010; 11(2):43-50.

11. Duarte LR, Silva DSJRd, Cardoso SH. Construindo um programa de educação com agentes comunitários de saúde. Interface. 2007;11(23):439-47.

12. BRASIL. Agência Nacional de Vigilância Sanitária ANVISA. Farmacopeia Brasileira. Memento Fitoterápico, 2016.

13. Franco LL. Doenças tratadas com plantas medicinais. 2.ed. Rio de Janeiro: Vozes, 2003.
14. Ghizi A, Mezzomo TR. Uso de plantas medicinais e satisfação de consumidores de lojas de produtos naturais do Mercado Municipal de Curitiba, PR. Revista Fitos; 2015;9(2):145-55.

15. Panizza ST, Veiga RS, Almeida MC. Uso tradicional de plantas medicinais e fitoterápicos.CONBRAFITO: São Luís, 2012.

16. Brasileiro GB, Pizziolo VR, Matos DS, Germano AM, Jamal CM. Plantas medicinais utilizadas pela população atendida no "Programa de Saúde da Família", Governador Valadares,MG, Brasil. Rev Bras Ciênc Farm. 2008; 44(4):629-36.

17. Pereira ZV, Mussury RM, Almeida AB, Sangalli A. Medicinal plants used by Ponta Porã community, Mato Grosso do Sul State. Acta Sci Biol Sci. 2009; 31(3):2939.

18. Jesus NZT, Lima JCS, Silva MM, Espinosa MM, Martins DTO. Levantamento etnobotânico de plantas popularmente utilizadas como antiúlceras e antiinflamatórias pela comunidade de Pirizal, Nossa Senhora do Livramento-MT, Brasil. Rev Bras Farmacogn . 2009;19(1A):130-39.

19. Associação Brasileira de Empresas de Pesquisa (ABEP). Critério de Classificação Econômica Brasil. Critério Brasil 2015 e atualização da distribuição de classes para 2016. Associação Brasileira de Empresas de Pesquisa, 2015.

20. Ethur LZ, Jobim JC, Ritter JG, Oliveira G, Trindade BS. Comércio formal e perfil de consumidores de plantas medicinais e fitoterápicos no município de Itaqui - RS. Rev Bras Plantas Med. 2011;13(2):121-8.

21. BRASIL. Agência Nacional de Vigilância Sanitária ANVISA. Farmacopeia Brasileira. Formulário Nacional Fitoterápico, 2010.

22. Veiga Junior VF, Pinto AC, Maciel MAM. Plantas medicinais: cura segura? Quím Nova. 2005;28(3):51928.

23. Brasil. Agênvia Nacional de Vigilância Sanitária Instrução Normativa $n^{\circ} 5$ de 11 de dezembro de 2008. Brasília, 2008.

23. Lima DF, Pereira DL, Franciscon FF, Reis C, Lima VS, Calvacanti PP. Conhecimento e uso de plantas medicinais por usuários de duas unidades básicas de saúde. Rev Rene. 2014;15(3):383-90.

24. Souza-Moreira TM, Salgado HR, Pietro R. O Brasil no contexto de controle de qualidade de plantas medicinais. Rev Bras Farmacog. 2010;20(3):435-40. 
25. Balbino EE, Dias MF. Farmacovigilância: um passo em direção ao uso racional de plantas medicinais e fitoterápicos. Rev Bras Farmacog. 2010;20(6):9921000 .

26. Silveira PFd, Bandeira MAM, Arrais PSD. Farmacovigilância e reações adversas às plantas medicinais e fitoterápicos: uma realidade. Rev Bras Farmacogn. 2008;18(4):618-26.

27. Borges RAM, Oliveira VB. Riscos associados ao uso de plantas medicinais durante o período da gestação: uma revisão. Rev Uniandrade. 2015;16(2):101-8.

\section{Correspondência}

Vinícius Bednarczuk de Oliveira

Rua Agostinho Brusamolin, 333, bloco 12, apto 508, Bairro CIC.

81312-090.

Curitiba - Paraná - Brasil

E-mail: vinicius.bednarczuk@hotmail.com 